\title{
AN OPERATOR SATISFYING DUNFORD'S CONDITION (C) BUT WITHOUT BISHOP'S PROPERTY $(\beta)$
}

\author{
by T. L. MILLER and V. G. MILLER
}

(Received 14 January, 1997)

\begin{abstract}
1. Introduction. For $X$ a complex Banach space and $U$ an open subset of the complex plane $\mathbb{C}$, let $\mathcal{O}(U, X)$ denote the space of analytic $X$-valued functions defined on $U$. This is a Fréchet space when endowed with the topology of uniform convergence on compact subsets, and the space $X$ may be viewed as simply the constants in $\mathcal{O}(U, X)$. Every bounded operator $T$ on $X$ induces a continuous mapping $T_{U}$ on $\mathcal{O}(U, X)$ given by $\left(T_{U} f(\lambda)=(\lambda-T) f(\lambda)\right.$ for every $f \in \mathcal{O}(U, X)$ and $\lambda \in U$. Corresponding to each closed $F \subset \mathbb{C}$ there is also an associated analytic subspace $X_{T}(F)=X \cap \operatorname{ran}\left(T_{\mathbb{C} / F}\right)$. For an arbitrary $T \in \mathcal{L}(X)$, the spaces $X_{T}(F)$ are $T$-invariant, generally non-closed linear manifolds in $X$.

An operator $T \in \mathcal{L}(X)$ has the decomposition property $(\delta)$ provided that the space $X$ decomposes as $X=X_{T}\left(\bar{U}_{1}\right)+X_{T}\left(\bar{U}_{2}\right)$ whenever $\left\{U_{1}, U_{2}\right\}$ is an open cover of the complex plane. $T \in \mathcal{L}(X)$ is decomposable in the sense of Foias provided that $T$ has property $(\delta)$ and that the analytic subspaces $X_{T}(F)$ are closed whenever $F$ is a closed subset of the plane; see [1], [3].

The local resolvent of $T$ at a vector $x \in X$ is the set $\rho_{T}(x)$ consisting of all $\lambda \in \mathcal{C}$ for which there is a open neighborhood $U$ such that $x \in T_{U} \mathcal{O}(U, X)$. The local spectrum of $T$ at $x$ is $\sigma_{T}(x)=\mathbb{C} \backslash \rho_{T}(x)$. If $T$ is such that for every closed $F \subset \mathbb{C}$ the linear manifold $\left\{x \in X: \sigma_{T}(x) \subset F\right\}$ is closed in $X$, then $T$ satisfies Dunford's condition $(C)$; see [4, XVI.1]. We say that $T$ has the single-valued extension property provided that $T_{U}$ is injective for every open $U \subset \mathbb{C}$; equivalently, if $X_{T}(F)=\left\{x \in X: \sigma_{T}(x) \subset F\right\}$ for every closed $F \subset \mathbb{C}$; see [6, Proposition 1.1].

An operator $T$ has Bishop's property $(\beta)$ provided that for every open $U \subset \mathbb{C}$ the mapping $T_{U}$ is injective and has closed range. Albrecht and Eschmeier [2] showed that property $(\beta)$ completely characterizes the restrictions of decomposable operators to invariant subspaces and their analytic functional model shows that every Banach space operator is similar to the quotient of an operator with property $(\beta)$; see [5]. Moreover, Albrecht and Eschmeier prove properties $(\beta)$ and $(\delta)$ to be completely dual; an operator $T$ has one of these precisely when $T^{*}$ has the other.

Property $(\beta)$ implies $(C)$, and $(C)$ in turn implies the single-valued extension property, [6, Proposition 1.2]. Therefore $T \in \mathcal{L}(X)$ has property $(C)$ if and only if the analytic subspaces $X_{T}(F)$ are closed whenever $F$ is a closed subset of the plane, and $T$ is decomposable if and only if $T$ has both properties $(C)$ and $(\delta)$; equivalently, if and only if $T$ has both properties $(\beta)$ and $(\delta)$. Thus it is a natural question whether property $(C)$ is strictly weaker than property $(\beta)$. Laursen and Neumann mention it explicitly in [6], but this question has circulated informally for some time.
\end{abstract}

ExAmPLE. Weighted shifts have proven to be a rich source of examples and are a favorite testing ground for operator theorists. The basic facts regarding weighted shifts are collected in Allen Shields' excellent survey [11].

Glasgow Math. J. 40 (1998) 427-430. 
Let $\alpha=\left(\alpha_{n}\right)_{n \geq 0}$ be a sequence of strictly positive real numbers with $\sup _{n \leq 0} \alpha_{n+1} / \alpha_{n}<\infty$, and consider the Hilbert space $H^{2}(\alpha)$, consisting of formal power series $f(z)=\sum_{n=0}^{\infty} \hat{f}(n) z^{n}$
satisfying

$$
\|f\|_{\alpha}^{2}:=\sum_{n=0}^{\infty}|\hat{f}(n)|^{2} \alpha_{n}^{2}<\infty
$$

An injective unilateral weighted shift can be realized as multiplication by the independent variable on a space $H^{2}(\alpha)$ for some $\alpha$ : let $T: H^{2}(\alpha) \rightarrow H^{2}(\alpha)$ be given by $(T f)(z)=$ $\sum_{n=0}^{\infty} \hat{f}(n) z^{n+1}$. Shields describes the spectrum of $T$ in terms of quantities $r, r_{1}$ and $r_{2}$, where

$$
r(\alpha)=\lim _{n \rightarrow \infty}\left(\sup _{k \geq 0} \frac{\alpha_{n+k}}{\alpha_{k}}\right)^{1 / n}, r_{1}(\alpha)=\lim _{n \rightarrow \infty}\left(\inf _{k \geq 0} \frac{\alpha_{n+k}}{\alpha_{k}}\right)^{1 / n} \text { and } r_{2}(\alpha)=\liminf _{n \rightarrow \infty} \alpha_{n}^{1 / n} .
$$

Clearly for any $\alpha$ we have $r_{1} \leq r_{2} \leq r$. By [11, Theorems 4 and 6], the shift $T$ has norm $\sup _{n \geq 0} \alpha_{n+1} / \alpha_{n}$, spectrum $\sigma(T)=\{z:|z| \leq r\}$ and approximate point spectrum given by $\sigma_{a p}(\bar{T})=\left\{z: r_{1} \leq|z| \leq r\right\}$. The point spectrum, $\sigma_{p}(T)$, is empty, and by [11, Theorem 8]

$$
\{0\} \cup\left\{z:|z|<r_{2}\right\} \subset \sigma_{p}\left(T^{*}\right) \subset\left\{z:|z| \leq r_{2}\right\} .
$$
Moreover by [11, Theorem 10], if $|\lambda|<r_{2}$, then the vector $k_{\lambda}(z)=\sum_{n=0}^{\infty} \bar{\lambda}^{n} \alpha_{n}^{-2} z^{n}$ is in
$\operatorname{ker}(\lambda-T)^{*}$ and is the reproducing kernel for evaluation at $\lambda$ :

$$
\left\langle f, k_{\lambda}\right\rangle_{H^{2}(\alpha)}=\sum_{n=0}^{\infty} \hat{f}(n) \lambda^{n}
$$

In particular, if $r_{2}>0$ then each $f \in H^{2}(\alpha)$ is analytic on the disk $\left\{z:|z|<r_{2}\right\}$.

Proposition. With the notation above, we have the following results. (1) If $r_{2}=r$, then $T$ satisfies Dunford's condition $(C)$; in fact, $\sigma_{T}(f)=\sigma(T)$ for every nonzero $f$ in $H^{2}(\alpha)$. (2) If $r_{1}<r_{2}$, then $T$ does not have Bishop's property $(\beta)$.

Proof. If $r=0$, then $T$ is quasiinilpotent and therefore decomposable. Thus we may assume that $r>0$. In this case, the first statement follows from [8, Proposition 1], but this is not hard to show directly. Indeed, if $f \in H^{2}(\alpha)$ is such that $\sigma_{T}(f) \neq \sigma(T)$ then there is an open subset $U$ of $\left\{z:|z|<r_{2}\right\}$ and a function $\varphi \in \mathcal{O}\left(U, H^{2}(\alpha)\right)$ such that $f=T_{U} \varphi$. For every $\lambda, \omega \in U$ we have that

$$
\begin{aligned}
f(\omega) & =\left\langle(\lambda-T) \varphi(\lambda), k_{\omega}\right\rangle=\left\langle\varphi(\lambda),(\lambda-T)^{*} k_{\omega}\right\rangle \\
& =(\lambda-\omega)\left\langle\varphi(\lambda), k_{\omega}\right\rangle
\end{aligned}
$$

In particular $f(\lambda)=0$, for every $\lambda \in U$, and since $f$ is analytic it follows that $f=0$.

To show the second assertion, suppose that $r_{1}<r_{2}$ and let $D$ be the disk $D=\left\{z:|z|<r_{2}\right\}$. If $\varphi \in \mathcal{O}\left(D, H^{2}(\alpha)\right)$ and $\lambda \in D$ let us write the power series for $\varphi(\lambda)$ as $\varphi(\lambda, z)=\sum_{n=0}^{\infty} \widehat{\varphi(\lambda)}(n) z^{n}$. 
For every $\lambda \in D$, let $H_{\lambda}=k_{\lambda}^{\perp}=\left\{f \in H^{2}(\alpha): f(\lambda)=0\right\}$. Notice that the polynomials in $H_{\lambda}$ are dense in $H_{\lambda}$; in fact,

$$
\{p: p \text { a polynomial, } p(\lambda)=0\} \subset(\lambda-T) H^{2}(\alpha) \subset H_{\lambda}
$$

and $\operatorname{ran}(\lambda-T)=H_{\lambda}$ only if $|\lambda|<r_{1}$. Choose $\lambda_{0}$ in $D$ with $\left|\lambda_{0}\right| \geq r_{1}$ and $f \in H_{\lambda_{0}} \backslash \operatorname{ran}\left(\lambda_{0}-T\right)$. Let $\left(p_{n}\right)_{n \geq 1}$ be a sequence of polynomials in $H_{\lambda_{0}}$ converging to $f$ in $H^{2}(\alpha)$, and define $\varphi$ and $\left(\varphi_{n}\right)_{n \geq 1}$ in $\mathcal{O}\left(D, H^{2}(\alpha)\right)$ by $\varphi(\lambda)=\left\langle f, k_{\lambda}\right\rangle 1-f$, and for each $n, \varphi_{n}(\lambda)=\left\langle p_{n}, k_{\lambda}\right\rangle 1-p_{n}$; that is, $\varphi(\lambda, z)=f(\lambda)-f(z)$, and $\varphi_{n}(\lambda, z)=p_{n}(\lambda)-p_{n}(z)$ for every $\lambda, z \in D$. Notice that $\varphi \notin \operatorname{ran}\left(T_{D}\right)$ since $f=-\varphi\left(\lambda_{0}\right)$ is not in $\operatorname{ran}\left(\lambda_{0}-T\right)$. Also, for every $\lambda \in D$ and for each $n$, there is a function $q_{n}$ such that $q_{n}(\lambda, z)$ is a polynomial in $z$, analytic in $\lambda$ and $\varphi_{n}(\lambda, z)=(\lambda-z) q_{n}(\lambda, z)$. In particular, each $\varphi_{n} \in \operatorname{ran}\left(T_{D}\right)$.

If $K$ is a compact subset of $D$, there is a positive constant $M$ such that $\left\|k_{\lambda}\right\|_{\alpha} \leq M$, for each $\lambda \in K$, and therefore

$$
\sup _{\lambda \in K}\left\|\varphi_{n}(\lambda)-\varphi(\lambda)\right\|_{\alpha} \leq \sup _{\lambda \in K}\left(\left|\left\langle p_{n}-f, k_{\lambda}\right\rangle\right|+\left\|p_{n}-f\right\|_{\alpha}\right) \leq(M+1)\left\|p_{n}-f\right\|_{\alpha} .
$$

It follows that $\varphi_{n} \rightarrow \varphi$ in $\mathcal{O}\left(D, H^{2}(\alpha)\right)$, and thus $T_{D}$ fails to have closed range.

To obtain the desired example, it remains only to construct an appropriate sequence $\alpha$. Let $\left(v_{j}\right)_{j=0}^{\infty}$ be a sequence of nonnegative integers with $v_{0}=0$, satisfying $v_{j}>v_{j-1}+j-1$ and

$$
\prod_{\ell=1}^{j}(1+\ell)^{-\ell / v_{j}}>\frac{j}{1+j}
$$
for each $j \geq 1$. Define the sequence $\alpha$ by $\alpha_{n}=1$, for $0 \leq n \leq \nu_{1}$, and $\alpha_{n}=\prod_{\ell=1}^{j}(1+\ell)^{-\ell}$ if
$v_{i}+j \leq n \leq v_{j+1}+j$.

COROllary. Let $\alpha$ be the sequence defined above and let $T$ be the injective unilateral weighted shift $T f(z)=z f(z)$, for $f \in H^{2}(\alpha)$. Then $r_{1}(\alpha)=0$ and $1=r_{2}(\alpha)=r(\alpha)=\|T\|$. In particular, $T$ has Dunford's property $(C)$ but not Bishop's property $(\beta)$.

Proof. Clearly $\|T\|=\sup _{n} \alpha_{n+1} / \alpha_{n}=1$; in fact, for each $n \geq 1$, if $\ell>n$, then $\alpha_{n+v_{\ell}} / \alpha_{\nu_{\ell}}=1$. Thus $1 \geq\left\|T^{n}\right\|=\sup _{k \geq 0} \alpha_{n+k} / \alpha_{k} \geq 1$, and $r(\alpha)=\|T\|=\overline{1}$.

For every $j \geq 1$,

$$
\left(\alpha_{j+v_{j}} / \alpha_{v_{j}}\right)^{1 / j}=\left(\frac{\prod_{\ell=1}^{j}(1+\ell)^{-\ell}}{\prod_{\ell=1}^{j-1}\left(1+\ell^{-\ell}\right)}\right)^{1 / j}=\frac{1}{j+1}
$$

it follows that $r_{1}(\alpha)=0$.

Finally, if $n \geq 1$ and if $v_{j}+j \leq n<v_{j+1}+j+1$, then

$$
\alpha_{n}^{1 / n}=\prod_{\ell=1}^{j}(1+\ell)^{-\ell / n} \geq \prod_{\ell=1}^{j}(1+\ell)^{-\ell / v_{j}}>\frac{j}{j+1}
$$

and therefore $\liminf _{n} \alpha_{n}^{1 / n} \geq 1$.

REMARKS. It follows, for example, from [7, Theorem 2] that an injective unilateral weighted shift $T$ has the decomposition property $(\delta)$ if and only if $\sigma(T)=\{0\}$, and in this case $T$ is decomposable. If the sequence $\left(\alpha_{n+1} / \alpha_{n}\right)_{n \geq 0}$ is increasing [11, Section 7], then the corresponding shift $T$ is hyponormal, and therefore has property $(\beta)$ by [9]. By [10, Corollary 2], if 
the sequence $\left(\alpha_{n+1} / \alpha_{n}\right)_{n \geq 0}$ is convergent with limit $r$, then the corresponding shift has property $(\beta)$ if $\left(r-\alpha_{n+1} / \alpha_{n}\right)_{n \geq 0} \in \ell^{p}$ for some $p$, with $1 \leq p<\infty$. Other than these cases, we do not know which shifts have Bishop's property $(\beta)$.

\section{REFERENCES}

1. E. Albrecht, On decomposable operators, Integral Equations Operator Theory, 2 (1979) 1-10.

2. E. Albrecht and J. Eschmeier, Analytic functional models and local spectral theory, preprint.

3. E. Albrecht, J. Eschmeier and M. Neumann, Some topics in the theory of decomposable operators, in Operator Theory: Advances and Applications, Vol. 17 (Birkhäuser, Basel, 1986), 15-34.

4. N. Dunford and J. T. Schwartz, Linears operators, Vol. III (Wiley-Interscience, New York, 1971).

5. K. B. Laursen, V. G. Miller and M. M. Neumann, Local spectral properties of commutators, Proc. Edinburgh Math. Soc. (2) 38 (1995), 313-329.

6. K. Laursen and M. Neumann, Asymptotic intertwining and spectral inclusions on Banach spaces, Czechoslovak Math. J. 43, (1993), 483-497.

7. T. Miller and V. Miller, Local spectral theory and orbits of operators, preprint.

8. T. Miller, V. Miller and R. Smith, The Cesàro operator and Bishop's property, J. London Math. Soc. (to appear).

9. M. Putinar, Hyponormal operators are subscalar, J. Operator Theory 12 (1984), 385-395.

10. M. Radjibalipour and H. Radjavi, On decomposability of compact perturbations of normal operators, Canada, J. Math. 27 (1975), 725-735.

11. A. L. Shields, Weighted shift operators and analytic function theory, Topics in Operator Theory (C. Pearcy, ed.), Mathematical Surveys 13 (AMS, Providence, RI, 1974).

Department of Mathematics

MisSissipi State University

Drawer MA

Mississippi STATE 39762

USA 